\title{
REVISED dot-app: a Graphviz-Cytoscape conversion plug-in
}

\section{[version 2; peer review: 4 approved]}

\author{
Braxton Fitts ${ }^{1}$, Ziran Zhang1 ${ }^{1}$ Massoud Maher ${ }^{1}$, Barry Demchak (iD)2 \\ ${ }^{1}$ Department of Computer Science and Engineering, UC San Diego, San Diego, USA \\ ${ }^{2}$ Department of Health Sciences, UC San Diego, San Diego, USA
}

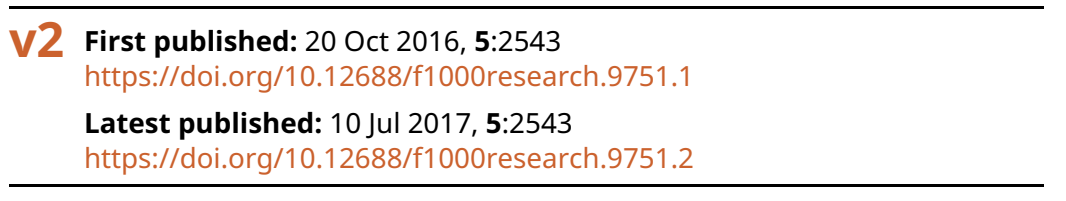

\section{Abstract}

dot-app is a Cytoscape 3 app that allows Cytoscape to import and export Graphviz (.dot, .gv) files, also known as DOT files due to the .dot extension and their conformance to the DOT language syntax. The DOT format was originally created in the early 2000 s to represent graph topologies, layouts and formatting. DOT-encoded files are produced and consumed by a number of open-source graph applications, including Graphviz, Gephi, Tulip, and others. While DOTbased graph applications are popular, they emphasize general graph layout and styling over the topological and semantic analysis functions available in domain-focused applications such as Cytoscape. While domain-focused applications have easy access to large networks (10,000 to 100,000 nodes) and advanced analysis and formatting, they do not have as many styling options as the Graphviz software suite. dot-app enables the interchange of networks between Cytoscape and DOT-compatible applications so that users can benefit from the features of both. dot-app was first deployed to the Cytoscape App Store in August 2015, has since registered more than 1,200 downloads, and has been highly rated by more than 20 users.

\section{Keywords}

Network, import, export, format conversion, attribute conversion , data visualization, Cytoscape , GraphViz , DOT

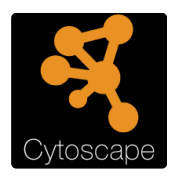

This article is included in the Cytoscape gateway.

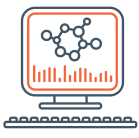

This article is included in the Bioinformatics

gateway.

\section{Open Peer Review}

$\begin{array}{rrrr}\text { Approval Status } & \checkmark \\ 1 & 2 & 3 & 4\end{array}$

version 2

(revision)

10 Jul 2017

version 1

20 Oct 2016
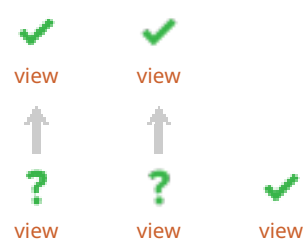

1. Eric Bonnet ID), CEA (French Alternative Energies and Atomic Energy Commission), Evry, France

2. Matthias König (iD), Humboldt University of Berlin, Berlin, Germany

3. David J. Lynn, South Australian Health and Medical Research Institute, Adelaide,

Australia

John Salamon, South Australian Health and Medical Research Institute, Adelaide, Australia

4. Giovanni Scardoni, University of Verona, Verona, Italy

Any reports and responses or comments on the article can be found at the end of the article. 
Corresponding authors: Braxton Fitts (bdtfitts@outlook.com), Ziran Zhang (ziz025@ucsd.edu), Massoud Maher( mahermassoud@gmail.com), Barry Demchak (idekerlab.bdemchak@gmail.com)

Author roles: Fitts B: Software, Validation, Visualization, Writing - Original Draft Preparation, Writing - Review \& Editing; Zhang Z: Software, Validation, Writing - Original Draft Preparation, Writing - Review \& Editing; Maher M: Software, Validation, Writing - Original Draft Preparation, Writing - Review \& Editing; Demchak B: Project Administration, Resources, Supervision, Writing - Original Draft Preparation, Writing - Review \& Editing

Competing interests: No competing interests were disclosed.

Grant information: This material is based upon work supported by the National Institutes of Health under Grant P41 GM103504. The grant is assigned to Dr. Trey Ideker at the University of California, San Diego (UCSD). All work was performed at UCSD. The funders had no role in study design, data collection and analysis, decision to publish, or preparation of the manuscript.

Copyright: (c) 2017 Fitts B et al. This is an open access article distributed under the terms of the Creative Commons Attribution License, which permits unrestricted use, distribution, and reproduction in any medium, provided the original work is properly cited.

How to cite this article: Fitts B, Zhang Z, Maher M and Demchak B. dot-app: a Graphviz-Cytoscape conversion plug-in [version 2; peer review: 4 approved] F1000Research 2017, 5:2543 https://doi.org/10.12688/f1000research.9751.2

First published: 20 Oct 2016, 5:2543 https://doi.org/10.12688/f1000research.9751.1 


\section{REVISED Amendments from Version 1}

With this version, we update several sections of the paper in order to clarify points and add further detail.

When we introduce Graphviz we include Table 1 that shows some graph software and their support for DOT files. We elaborate on our discussion of the visual features that the DOT file format support in comparison to other file formats which Cytoscape supports. Instead of comparing DOT to XGMML and GraphML, in a new Table 2 we compare DOT to XGMML and GEXF because GEXF includes visual features in its specification similar to DOT and XGMML.

An Installation subsection is added to the Operation section. To clarify how we used JPGD, we reworded the Import subsection of the Implementation. We state the unit conversion made when converting a node's height and weight values between Cytoscape and DOT.

In our Supplementary Material we include a document listing the DOT attributes ignored during import (Supplementary File 1), a ZIP file containing files from our Use Cases (Supplementary File 2), and a ZIP file containing all of our DOT files and Cytoscape session files that we used for testing (Supplementary File 3)

We also updated our software to use DOT default values when importing a network, and updated all of our images in the Use Cases section to reflect that change. Figure 1 is new, and Figure 12, Figure 13 and Figure 14 are revised form Version 1's Figures 11, 12 and 13 , respectively.

See referee reports

\section{Introduction}

Cytoscape $^{1}$ is a popular tool for visualizing and analyzing networks used in scientific and commercial analysis, most commonly in bioinformatics. It enables users to discover and load curated and uncurated networks representing molecular and genomic interactions, load ad-hoc or custom networks, and share networks that others have created. Once networks are loaded, users can manually annotate a network or automatically integrate annotations using a number of algorithms and databases. Users can perform a number of graph-oriented and semantic-aware analyses ranging from graph statistics to motif and cluster discovery to upstream and downstream structural and functional inferences. Users can also perform a number of complex graph filtering and layout operations to drive and focus the semantic understanding of network interactions and structure.

Even beyond analysis and layout, users commonly derive and demonstrate network meaning by using visual cues to distinguish relationships and attributes. For this, Cytoscape provides a visual style system that enables users to paint nodes and edges using color, border thickness, size, fonts, arrows, and other devices.

Much of the power and functionality of Cytoscape is delivered as apps available in the Cytoscape App Store (http://apps.cytoscape. org). The store contains 319 apps (June 2017) that provide a range of functionality from file import/export to analysis to visualization and publishing. Based on the success of the combination of Cytoscape core and downloadable apps, Cytoscape is downloaded approximately 14,000 times per month worldwide and is started approximately 3,000 times each working day. As of 2015, Cytoscape has been cited in 700 academic peer-reviewed papers per year.

While Cytoscape is the dominant network analysis and visualization platform in bioinformatics, it is not the only platform. To support interoperability with a number of network-oriented workflows and applications, Cytoscape offers a number of natively supported file import/export modules, and leverages a number of them that are available as apps in the App Store. Some of these file formats $^{2}$ do not include visual information, such as SIF (.sif) and NNF (.nnf). Other file formats, such as GEXF (.gexf) and XGMML(.xgmml), include varying amounts of visual information. Our primary motivation for creating dot-app was to extend Cytoscape's interoperability to users that primarily use Graphviz software. Prior to dot-app, a user would either have to convert the network to a file format compatible with Cytoscape or attempt to manually reconstruct the network. Converting a DOT file into a Cytoscape network by use of an intermediary file format is an inherently bad process because each conversion results in a loss of some data. Furthermore, reconstructing a graph manually in Cytoscape might not be too difficult for a simple graph with few nodes and edges, but reconstruction would become more time consuming for larger networks which have nodes of distinct visual properties since each node would have to be created one at a time and these visual properties have to be set separately for each node if there is no underlying data to which the visual properties can be mapped. With dot-app, we minimize information loss by providing a direct conversion from DOT to a Cytoscape network and we reduce the time needed to reconstruct the DOT network in Cytoscape.

Graphviz $^{3}$ is a popular, well-established graph visualization software suite that produces DOT (.dot, .gv) files containing graph structure, layout, and styling information; and uses these files to output these networks in a variety of file formats. These files adhere to the DOT language syntax (http://www.graphviz.org/doc/info/ lang.html). In this paper, we use "DOT file/network" and "Graphviz file/network" interchangeably. Due to Graphviz's continued usage, many graph visualization and analysis applications support the import and export of DOT files. Table 1 lists some of these applications and whether they can import or export DOT files.

As mentioned earlier, Cytoscape provides support for XGMML (http://www.cs.rpi.edu/research/groups/pb/punin/public_html/ XGMML/) and GEXF (http://www.gexf.net/format/index.html), which are two file formats that support visual information such as

Table 1. Some graph visualization and analysis software and their compatibility with DOT files.

\begin{tabular}{|l|l|l|}
\hline Graph Software & Import DOT files & Export DOT files \\
\hline Tulip & Supported & Not Supported \\
\hline Gephi & Supported & Not Supported \\
\hline NetworkX & Supported & Supported \\
\hline SocNetV & Supported & Not Supported \\
\hline
\end{tabular}


node shapes and line types. In comparison to GEXF and XGMML, the DOT format defines more node shapes, arrow shapes, and has unique features such as gradients for node colors and edges painted with multiple colors. Table 2 shows some options for edge line types and which file formats support them. GEXF's entry for multiple lines is marked with an asterisk because its support for the line type is limited. Unlike DOT which supports any number of lines for an edge, GEXF only supports up to two lines for an edge with its "double" line type. Figure 1 is a graph of all the node shapes
Table 2. Edge line types available with DOT, XGMML, or GEXF. X's are supported types.

\begin{tabular}{|l|l|l|l|l|l|}
\hline $\begin{array}{l}\text { Line Types/ } \\
\text { File Format }\end{array}$ & Solid & Dotted & Dashed & Tapered & Multiple \\
\hline DOT & $\mathbf{X}$ & $\mathbf{X}$ & $\mathbf{X}$ & $\mathbf{X}$ & $\mathbf{X}$ \\
\hline GEXF & $\mathbf{X}$ & $\mathbf{X}$ & $\mathbf{X}$ & & $\mathbf{X}^{\star}$ \\
\hline XGMML & $\mathbf{X}$ & & $\mathbf{X}$ & & \\
\hline
\end{tabular}

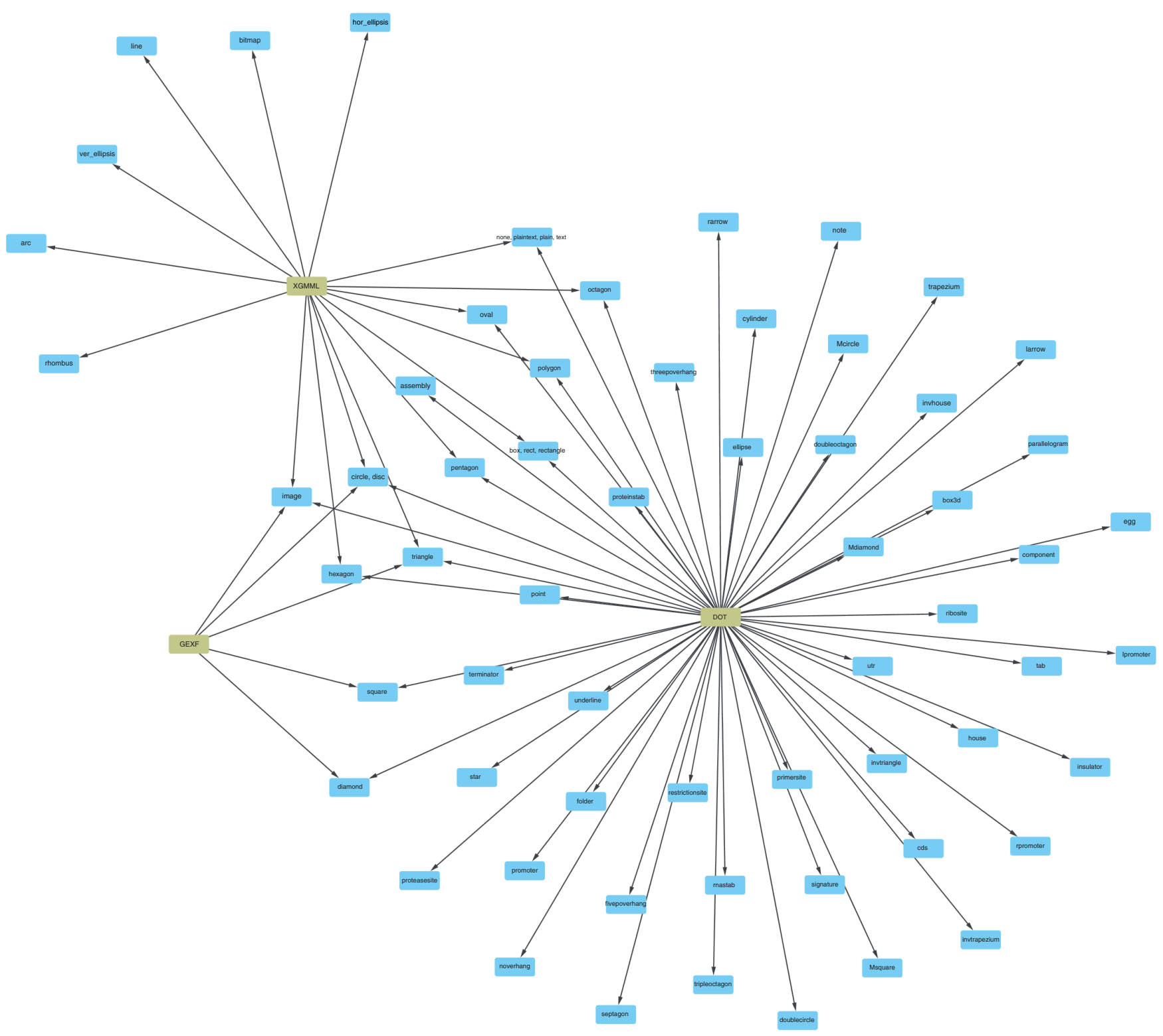

Figure 1. Graph showing which node shapes are supported by which file format. 
between the three formats and shows which formats support which node shapes. Names for the same node shape share a node in the graph, such as the "disc" shape name found in GEXF and the "circle" shape name found in both DOT and XGMML.

DOT files contain a number of visual attributes that map well to Cytoscape visualization functionality, and vice versa. However, incompatibilities do exist where some Cytoscape features cannot be represented in DOT, or where DOT represents some features that cannot be realized in Cytoscape. These incompatibilities are described in "Conversion details" section.

We present dot-app as a Cytoscape app that implements both the import and export of graphs encoded in DOT files. We describe the operation of dot-app, how dot-app maps Cytoscape networks to DOT networks and vice versa, issues that arise because of incompatibilities between the Cytoscape and DOT network models, representative use cases, and prospects for future work.

\section{Operation}

Minimum system requirement

Dot-app requires Java 7 or above and Cytoscape v3.2 or above.

\section{Installation}

Open Cytoscape and navigate to the App Manager from the menu ("Apps->App Manager"). While in the "Install Apps" tab, type dotapp in the search bar. Make sure the download site is http://apps. cytoscape.org, as our app is in the Cytoscape App Store. Click on dot-app to select it and then click the install button to download and install the app.
Import

A Graphviz network can be imported in three ways: from the welcome screen (via the "From Network File..." button), from the menu ("File->Import->Network->File..."), or from the toolbar (by clicking the "Import Network from File" button).

Users are presented with a file browser dialog titled "Network file to load" (as in Figure 2). The user is able to filter the dialog to display only Graphviz files by selecting "GraphViz files (*.gv, *.dot)" from the drop-down menu for "Files of Type". Note that no difference exists between a Graphviz file with an extension of dot and a Graphviz file with an extension of .gv. However, the .gv extension is preferred because versions of Microsoft Word also use the .dot extension (https://marc.info/?l=graphviz-devel\&m=1294 18103126092). From this point, importing a Graphviz network is the same as importing a network from any of Cytoscape's accepted file formats. Those steps are detailed in the Cytoscape User Manual (http://manual.cytoscape.org/en/stable/).

\section{Export}

To export a Cytoscape network as a Graphviz network, use "Export -> Network and View" from the menu. (Using "Export -> Network" is also possible, but this will result in a Graphviz file that contains no visual information and a notification to use "Export -> Network and View" instead.)

Selecting "GraphViz files (*.dot,*.gv)" in the Export dialog launches dot-app and prompts the user to choose from three options, as shown in Figure 3 below. The purposes of these options are explained in the following section.

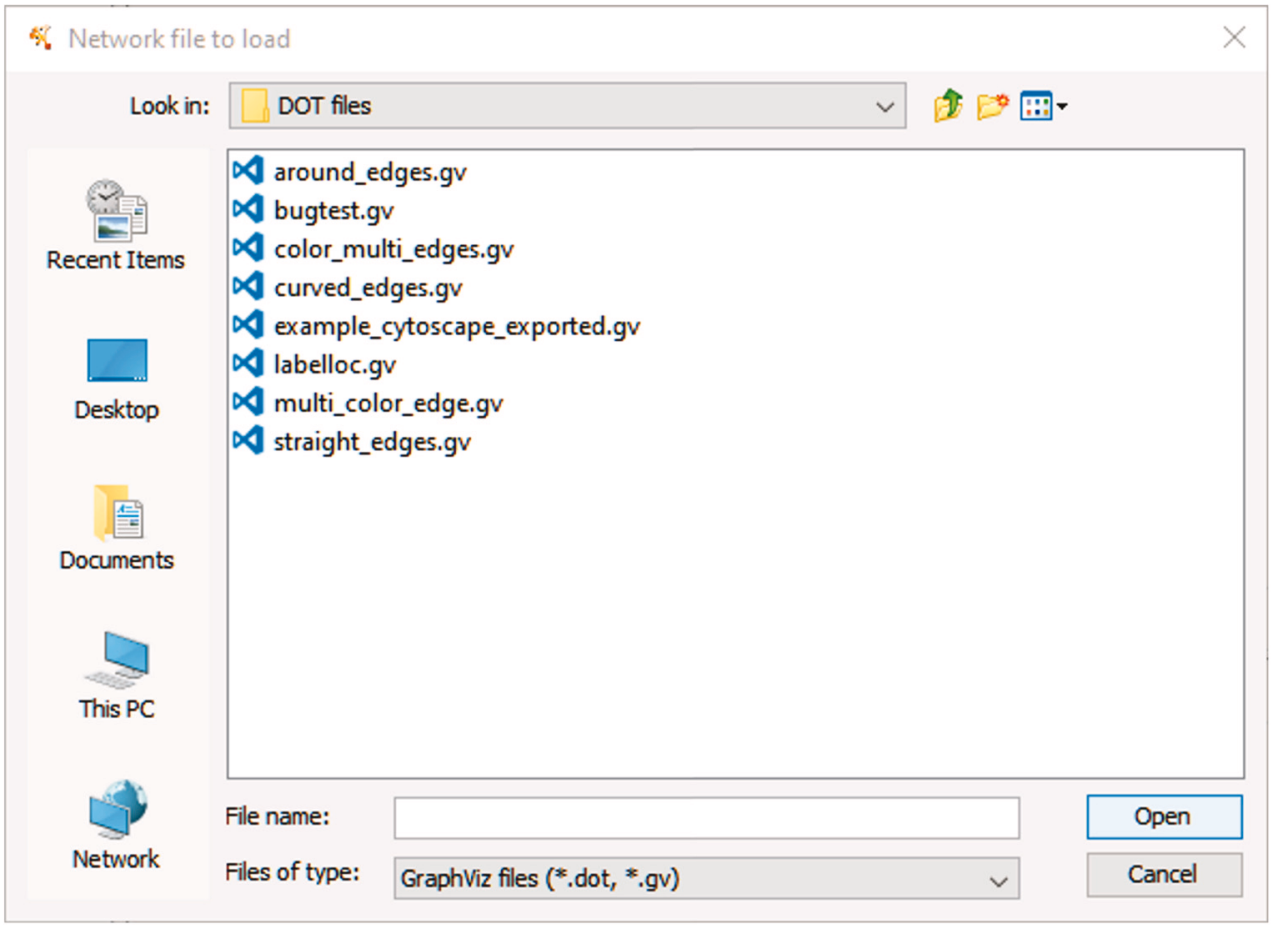

Figure 2. Network file to load dialog with Graphviz files selected. 


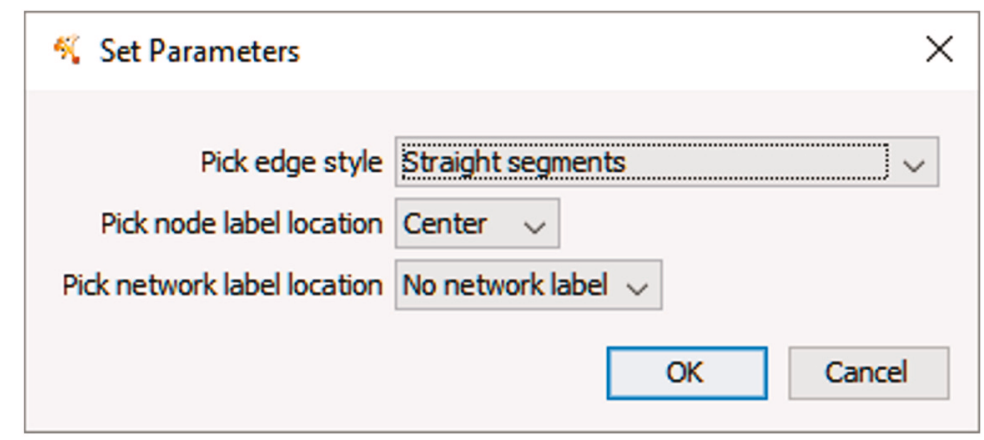

Figure 3. Set Parameter pop-up prompts users with three options.

\section{Set Parameters prompt}

Pick edge style. Cytoscape provides edge-routing capabilities that cannot be conserved during the export process, so dot-app provides three edge routing options: "Straight segments", "Curved segments", and "Curved segments routed around nodes". These options change the value of the "splines" attribute that appears in the exported Graphviz file. The Graphviz file for a network exported from Cytoscape is shown below, and the attribute modified by the "Pick Edge Style" option is underlined and in bold. Figure 4, Figure 5, and Figure 6 depict pictures of the network with each edge style chosen.

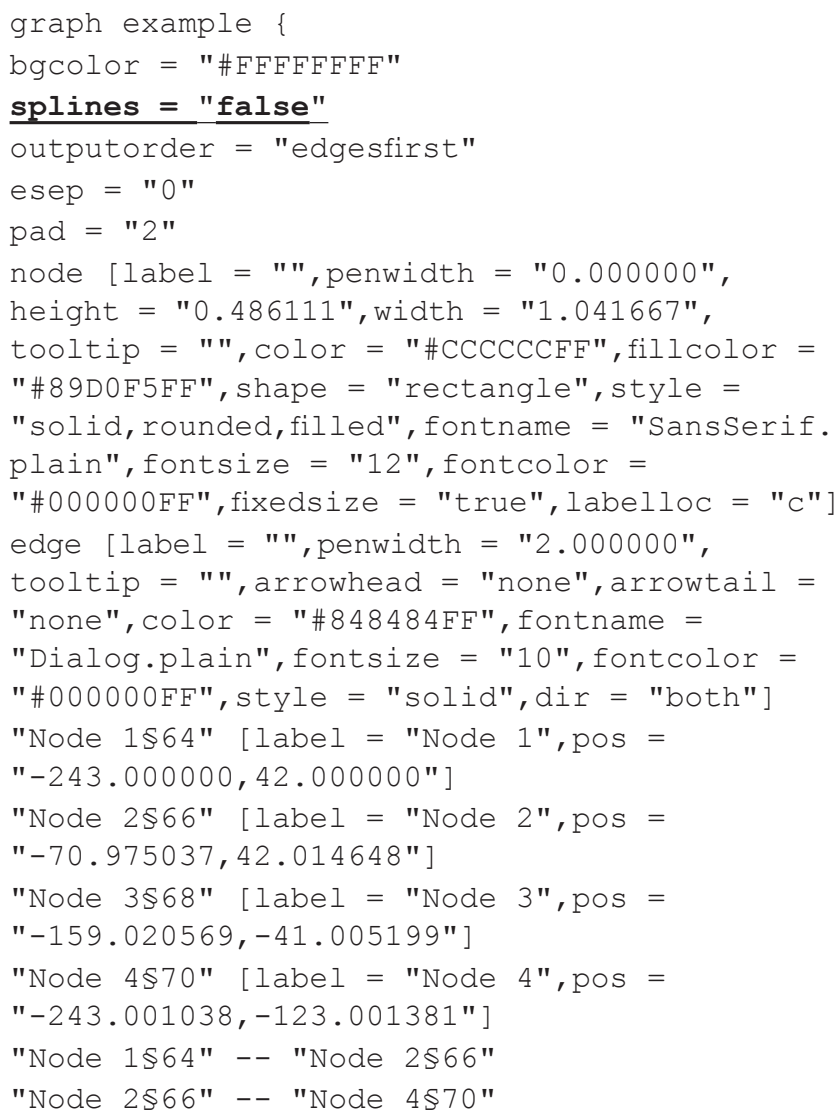

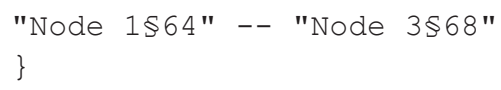

Pick node label location. Graphviz does not offer the flexible label placement that Cytoscape offers. As such, dot-app gives the options of "Center," "Top," "Bottom," and "External" to allow the user to specify the label location applied to every node. In the output Graphviz file, the "Center", "Top", and "Bottom" options change the value of the "labelloc" attribute that appears in the node default attribute list. The options respectively change the value to "c", " $t$ ", and "b". In contrast, the "External" option causes the node labels to set the "xlabel" attribute instead of the "label" attribute in the output Graphviz file. The "xlabel" attribute causes the label to be placed in a location near its node that does not cause it to overlap with any other nodes or labels. Figure 7 shows a network exported with the "External" option.

Pick network label location. dot-app provides the options "No network label," "Top," and "Bottom" to allow the user to specify whether the network itself should be labeled and, if so, where the label is placed. The options "Top" and "Bottom" cause the "labelloc" attribute and "label" attribute for the graph to be written to the output Graphviz file. Furthermore, the "label" attribute will be set to the network's name in Cytoscape. In contrast, the "No network label" option omits both the "labelloc" attribute and the "label" attribute.

\section{Implementation}

Import

For the import function of dot-app, we used Java-based Parser for Graphviz Documents (JPGD), a Graphviz document parser made by Alexander Merz (http://www.alexander-merz.com/graphviz/). We use JPGD to read the DOT file and create a JPGD data model of the graph or collection of graphs in the file. A JPGD Graph object is created for each graph in the file, a JPGD Node object for each node, and a JPGD Edge object for each edge. Each JPGD object contains the DOT attributes for the graph element they represent. Figure 8 provides a high-level picture of the conversion of a DOT node declaration to the JPGD Node object. Detailed information about the JPGD objects can be found on JPGD's website (http:// www.alexander-merz.com/graphviz/doc.html). 


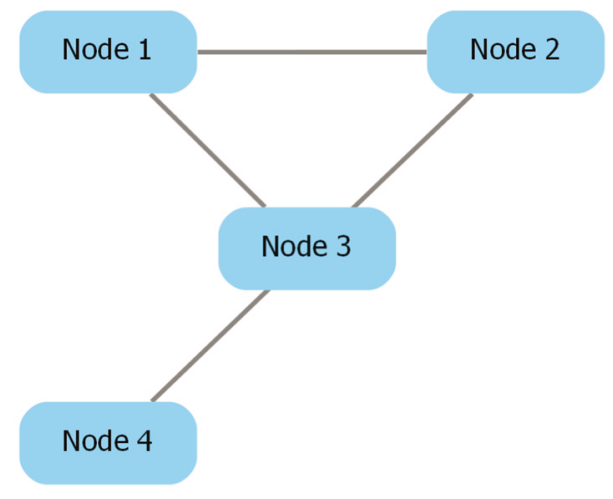

Figure 4. The network exported with the "Straight segments" option, as "splines = "false"”' in the output Graphviz file.

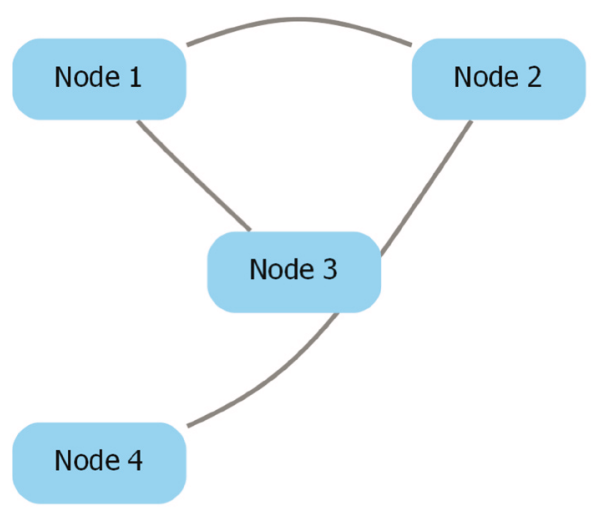

Figure 5. The network exported with the "Curved segments" option, as "splines = "curved" in the output Graphviz file.

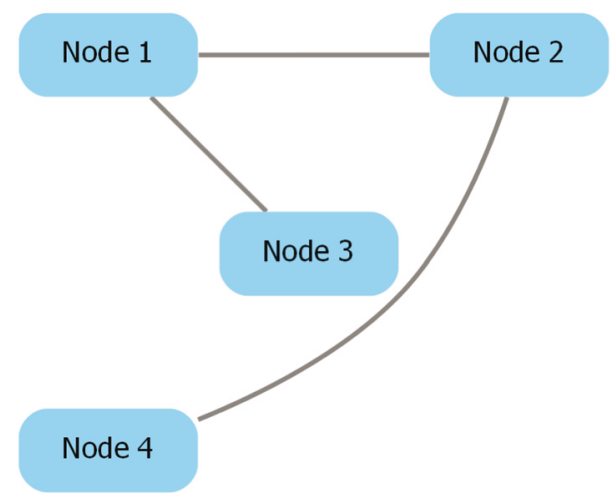

Figure 6. The network exported with the "Curved segments routed around nodes" option, as "splines = "true"' in the output Graphviz file.

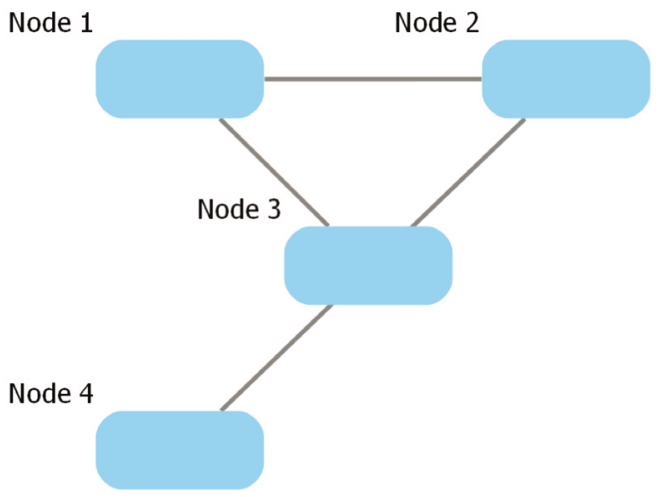

Figure 7. A network exported with the "External" option for node label location.

Once the JPGD data model is built, we begin creation of the network in Cytoscape by using the JPGD data model to create a Cytoscape data model. A CyNetwork object is created for each JPGD Graph object, a CyNode object for each JPGD Node object, and a CyEdge object for each JPGD Edge object. At this point in the importation, the graph elements themselves have been imported, but not their visual attributes.

A Cytoscape View data model has to be created in order for the DOT graphs to be visually recreated in Cytoscape. In order to facilitate this, we associate each JPGD object to their corresponding Cytoscape object and store these associations in maps. These maps are necessary because the Cytoscape View data objects are created from the Cytoscape objects, yet the visual attributes for each element is stored in the JPGD objects. When the network view is being built in Cytoscape, our Reader objects use these associations to create the Cytoscape View objects. Three Reader classes exist: NetworkReader, NodeReader, and EdgeReader. At the start of the network view creation, a VisualStyle object is created for the network. This VisualStyle is constructed so that if the DOT file does not specify default attributes then the default attributes are already set to DOT implicit values. Each Reader object uses the VisualStyle to set the default attributes for its class of graph components. In addition, each Reader iterates through their association map to retrieve the View objects for the graph components. After the View objects are retrieved, the DOT attributes and their assigned values are converted into their Cytoscape equivalents, and the resulting VisualProperty and VisualPropertyValue are set to the View. If a DOT attribute does not have an equivalent VisualProperty or its assigned value does not have an equivalent VisualPropertyValue then it is ignored. Figure 9 shows the highlevel relationships among the JPGD objects, the Cytoscape objects, and the Cytoscape View objects.

\section{Export}

We created three classes-NodePropertyMapper, EdgePropertyMapper and NetworkPropertyMapper-to accomplish the export function of dot-app. Each Mapper class contains an ArrayList into 


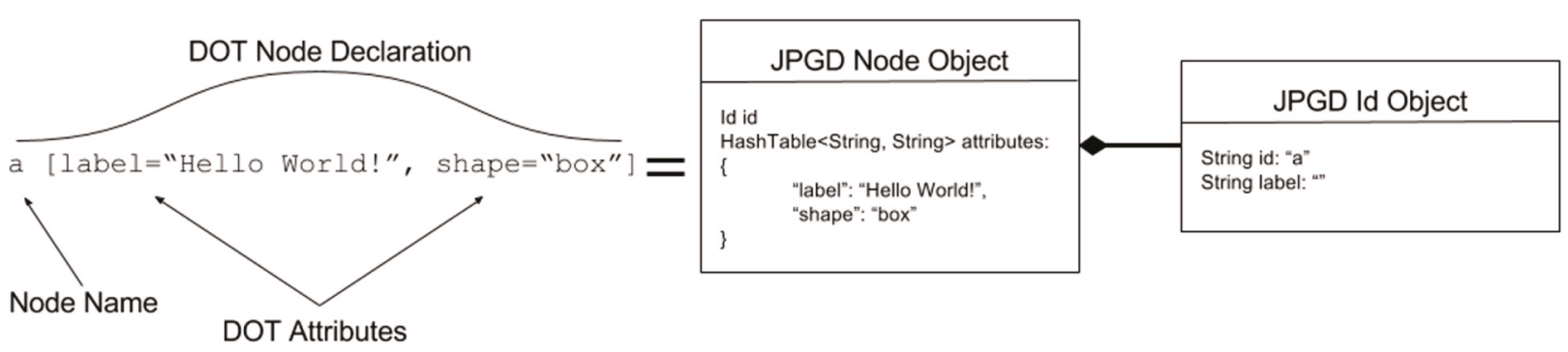

Figure 8. DOT Node Declaration to JPGD Node Object.

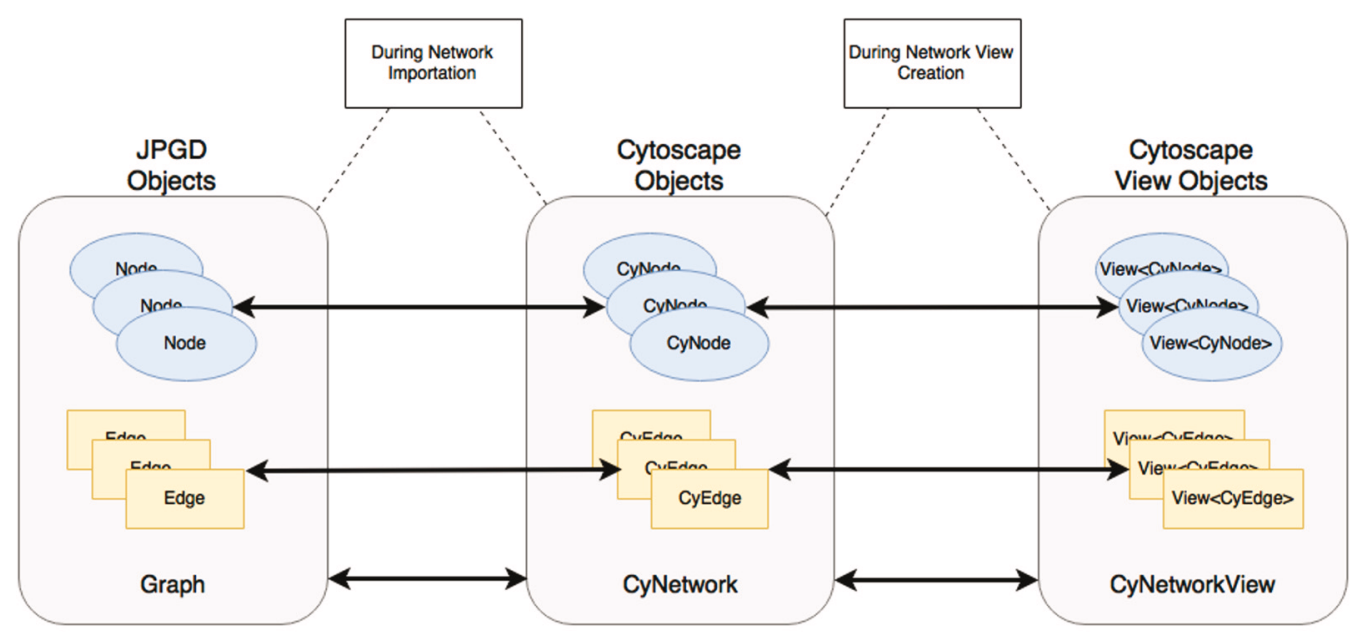

Figure 9. Relationships between JPGD objects, Cytoscape objects and Cytoscape view objects.

which the Mapper classes insert the DOT attribute strings for easily convertible Cytoscape VisualProperties. In addition, each Mapper class has unique helper methods that create the DOT attribute strings for the DOT attributes that have values determined by multiple Cytoscape VisualProperties. One such attribute is the "style" DOT attribute, the value of which is determined by NODE_ SHAPE, NODE_BORDER_LINE_TYPE, and NODE_VISIBLE. The NodePropertyMapper class handles the conversion of the CyNodes and their VisualProperties into their DOT string equivalents. The EdgePropertyMapper class handles the conversion of the CyEdges and their VisualProperties into their DOT string equivalents. Finally, the NetworkPropertyMapper class handles the conversion of the CyNodes' and CyEdges' default VisualProperties and the CyNetwork's VisualProperties into their DOT string equivalents. In order to build the final DOT attribute declaration string for each element, we concatenate all of the individual DOT attribute strings together.

\section{Conversion details}

Import

Supported DOT attributes. The DOT attributes in the following subsections contribute to the Cytoscape network during the import process. Most of the DOT attributes listed below correspond to a single Cytoscape visual property and their values are able to copied over easily (such as the label attribute), but a few of the attributes either affect multiple visual properties at once (e.g., the "style" DOT attribute, as described below) or a conversion needs to performed on their values (e.g., the "height" and "width" attributes) due to the fact that the information is stored differently between the DOT model and the Cytoscape model. If a DOT attribute is assigned a value that does not have an equivalent value for the corresponding Cytoscape visual property, then the DOT attribute is ignored. The "weight" DOT attribute is imported as an Edge table attribute (i.e., data) because no corresponding Cytoscape visual property exists. The DOT attributes listed in File 1 of the Supplementary Material are ignored and have no effect on the visualization in Cytoscape.

Node DOT attributes. Table 3 lists the DOT attributes that can apply to nodes and the specific Cytoscape visual properties to which they map. The "pos" attribute maps to both NODE_X_ POSITION and NODE_Y_POSITION because the value of the "pos" attribute is a coordinate pair of the form " $\mathrm{x}, \mathrm{y}$ ".

Edge DOT attributes. Table 4 lists the DOT attributes that apply to edges and the specific Cytoscape visual properties to which they map. 
The "style" DOT attribute. The "style" DOT attribute applies to both nodes and edges. The attribute takes a comma-separated list of keywords as its value. These keywords directly affect which Cytoscape visual properties are modified. Table 5 lists the keywords that dot-app supports, the graph components they affect and the Cytoscape visual properties to which the keywords map.

\section{Table 3. DOT node attributes and their corresponding Cytoscape visual properties.}

\begin{tabular}{|l|l|}
\hline DOT attribute & Cytoscape visual property \\
\hline label & NODE_LABEL \\
\hline xlabel & NODE_LABEL \\
\hline color & NODE_BORDER_PAINT \\
\hline fillcolor & NODE_FILL_COLOR \\
\hline penwidth & NODE_BORDER_WIDTH \\
\hline width & NODE_WIDTH \\
\hline height & NODE_HEIGHT \\
\hline shape & NODE_SHAPE \\
\hline fontname & NODE_LABEL_FONT_FACE \\
\hline fontsize & NODE_LABEL_FONT_SIZE \\
\hline fontcolor & NODE_LABEL_FONT_COLOR \\
\hline style & $\begin{array}{l}\text { See section "The 'style' DOT } \\
\text { Attribute” }\end{array}$ \\
\hline pos & NODE_X_POSITION, \\
\hline NODE_Y_POSITION \\
\hline tooltip & NODE_TOOLTIP \\
\hline
\end{tabular}

Table 4. DOT edge attributes and their corresponding Cytoscape visual properties.

\begin{tabular}{|l|l|}
\hline DOT attribute & Cytoscape visual property \\
\hline label & EDGE_LABEL \\
\hline xlabel & EDGE_LABEL \\
\hline weight & No Visual Property \\
\hline color & EDGE_UNSELECTED_PAINT \\
\hline penwidth & EDGE_WIDTH \\
\hline fontname & EDGE_LABEL_FONT_FACE \\
\hline fontsize & EDGE_LABEL_FONT_SIZE \\
\hline fontcolor & EDGE_LABEL_FONT_COLOR \\
\hline style & See section "The 'style' DOT \\
\hline arrowhead & Ettribute” \\
\hline arrowtail & EDGE_TARGET_ARROW_SHAPE \\
\hline tooltip & EDGE_TOOLTIP \\
\hline
\end{tabular}

Table 5. The keywords for the DOT style attribute, the graph element that it can affect, and the Cytoscape visual properties that are modified as a result (the "rounded" keyword only affects NODE_SHAPE if the shape="rectangle").

\begin{tabular}{|c|c|c|}
\hline $\begin{array}{l}\text { "Style" attribute } \\
\text { keyword }\end{array}$ & $\begin{array}{l}\text { Affects nodes } \\
\text { or edges }\end{array}$ & Cytoscape visual property \\
\hline solid & Nodes/Edges & $\begin{array}{l}\text { NODE_BORDER_LINE_TYPE } \\
\text { EDGE_LINE_TYPE }\end{array}$ \\
\hline dashed & Nodes/Edges & $\begin{array}{l}\text { NODE_BORDER_LINE_TYPE } \\
\text { EDGE_LINE_TYPE }\end{array}$ \\
\hline dotted & Nodes/Edges & $\begin{array}{l}\text { NODE_BORDER_LINE_TYPE } \\
\text { EDGE_LINE_TYPE }\end{array}$ \\
\hline invis & Nodes/Edges & $\begin{array}{l}\text { NODE_VISIBLE } \\
\text { EDGE_VISIBLE }\end{array}$ \\
\hline rounded & Nodes & NODE_SHAPE* \\
\hline filled & Nodes & NODE_TRANSPARENCY \\
\hline
\end{tabular}

The "weight" DOT attribute. During the import of a network using dot-app, a weight column is added to the Cytoscape network's edge table. If the "weight" attribute is supplied for an edge, its value is assigned to the weight column entry for the edge.

The "height" and "width" DOT attributes. In the DOT language, the height and width of nodes are specified in inches. However, the height and width of nodes are specified in points in Cytoscape. During both import and export we handle the necessary unit conversion ( 1 in $=72 \mathrm{pts}$ ) in order to maintain the correctness of the graph.

Unsupported DOT features. The following features of Graphviz are not supported in the import:

\section{HTML-like Labels}

2. All node shapes are not supported except the following: triangle, diamond, ellipse, hexagon, octagon, parallelogram, rectangle, rect, box, and square. Msquare, Mcircle, and Mdiamond will be rendered the same as square, circle, and diamond respectively.

3. All arrow shapes are not supported except the following: vee, lvee, rvee, dot, normal, diamond, none, and tee. The arrow shapes odot, onormal, and odiamond will be rendered the same as dot, normal, and diamond respectively.

4. Edge curves. The value of the "pos" attribute is ignored when it is used as an attribute for edges, so dot-app will render edges only as straight lines between nodes.

5. The brewer colors chemes when using the "colorscheme" attribute.

6. The "tapered" and the "bold" edge style keywords

7. The "bold", "diagonals", "striped", and "wedged" node style keywords 
8. Default attribute values when grouping nodes with subgraphs. Only the default attribute values set within the root graph are considered.

9. Record-based Nodes. The label will not cause the node to become sectioned

\section{Clusters}

11. Edges that are rendered as colored parallel lines: These are made by assigning a color list without weights to the "color" attribute. Figure 10 depicts an example edge rendered in this manner.

12. Edges that are rendered as colored segments in series. These are made by assigning a color list with weights to the "color" attribute. Figure 11 depicts an example edge rendered in this manner.

13. Gradients applied to the network background

\section{Export}

Unsupported Cytoscape features. When exporting the network as a GraphViz file, some Cytoscape information is lost because it

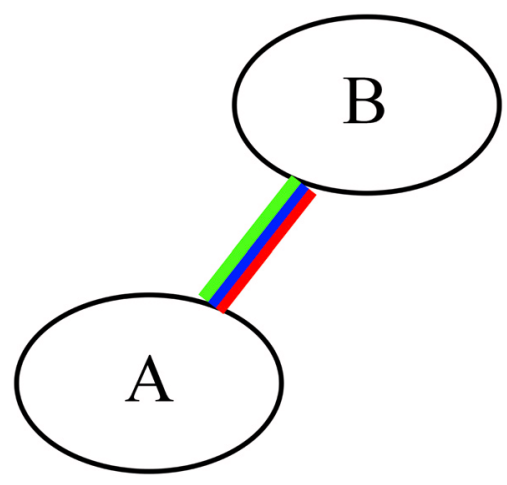

Figure 10. Example of an edge rendered as colored parallel lines.

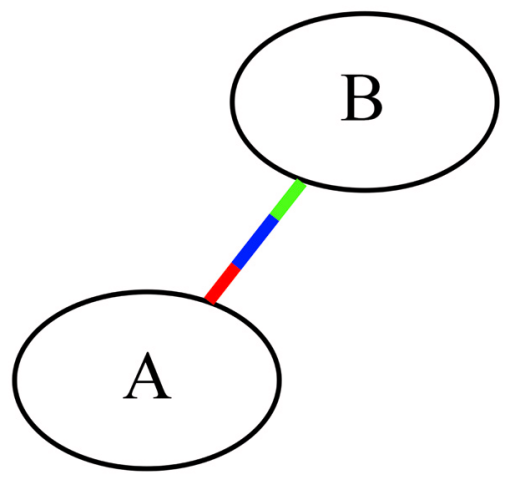

Figure 11. Example of an edge rendered as colored segments in series. cannot be represented in DOT format. dot-app does not keep a log of the information that is not transferred to the Graphviz file. The following information is lost:

\section{Visual information}

1. Custom graphics
a. Images on nodes
b. Charts on nodes

2. Edge bends

3. Nested network images contained in nodes

4. Arrowhead colors (they will appear the same color as the edge itself)

5. Certain line types
a. Dash dot
b. Contiguous arrow
c. Backward slash
d. Separate arrow
e. Sinewave
f. Vertical slash
g. Zigzag
h. Forward slash
i. Parallel lines

6. Label positioning

a. Edge labels only go on the midpoint of the edges

b. Node label positions are selected at export

7. The V node shape

8. Target arrow shape

a. Target arrow shape does not appear if set as a default (this was a bug in Cytoscape v3.2 that has been fixed since v3.3)

9. All annotations

\section{Non-visual information}

1. Node group information (groups that are collapsed are treated as a single node with no additional data)

\section{All table data}

\section{Use cases}

Detailed below are two cases for dot-app. The first use case describes how a DOT file can be imported into Cytoscape. The 
second use case describes how a Cytoscape network can be exported as a DOT file. The DOT file used for import, the Cytoscape session file of the network, and the DOT file generated by dot-app during export are contained in ZIP 1 of the Supplementary Material.

\section{Import}

Our first use case details how we would use dot-app to view a Graphviz-created network in Cytoscape. We used Graphviz's neato utility to create a DOT file with layout information and a PNG of the resulting network. The DOT file is shown below, and Figure 12 is the created PNG.
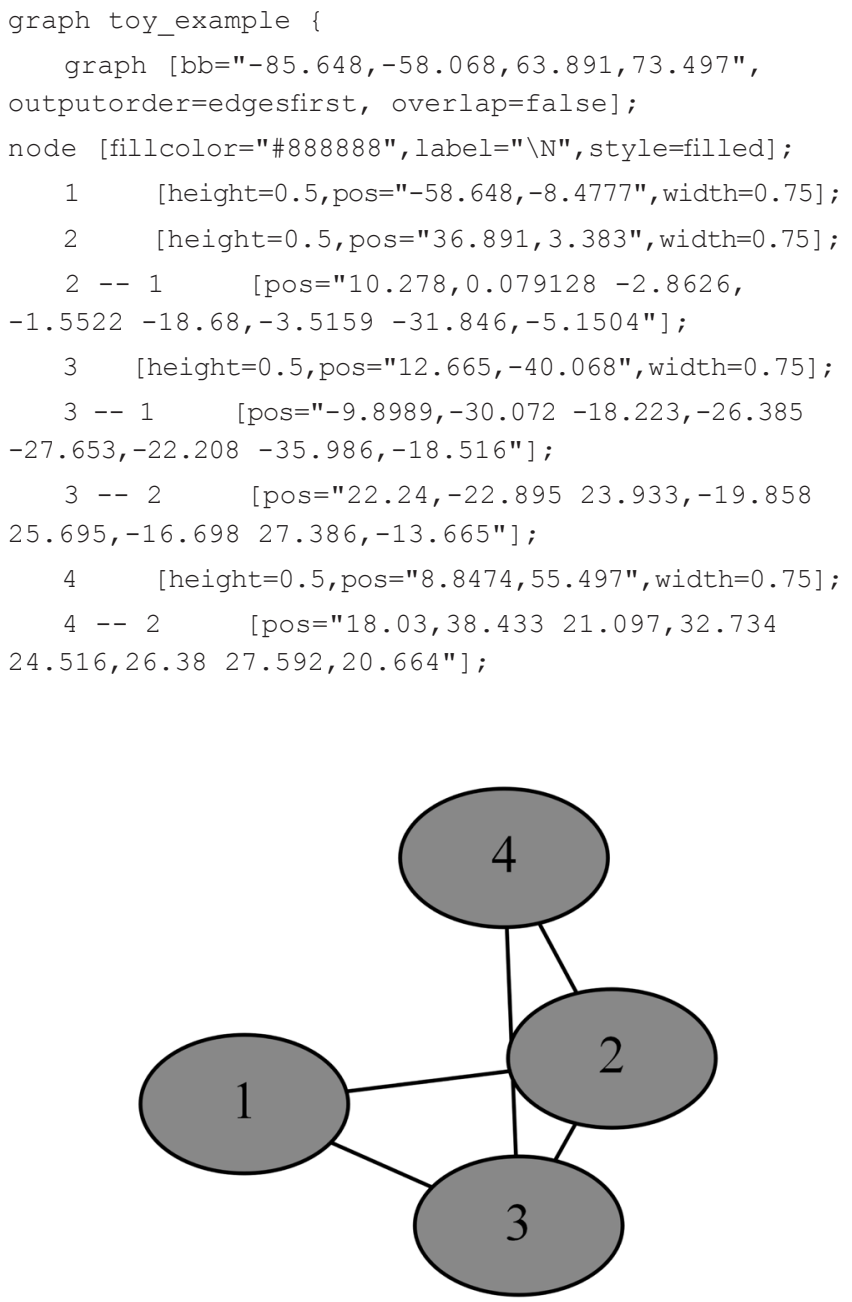

Figure 12. The PNG generated using Graphviz's neato utility.

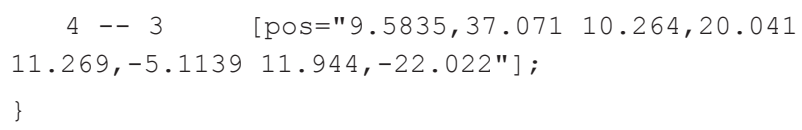

Figure 13 shows the result of the import into Cytoscape version 3.5.1. For a basic DOT file which uses attributes that have equivalents in Cytoscape, dot-app creates a faithful reproduction. A minor difference between the two is the width of the node borders. This is due to the difference between how Cytoscape and Graphviz render a node border with a width of 1 .

\section{Export}

In this second use case, we export the network in Figure 13 from Cytoscape. The output file is shown below. Figure 14 shows the PNG created by using Graphviz's neato utility on the

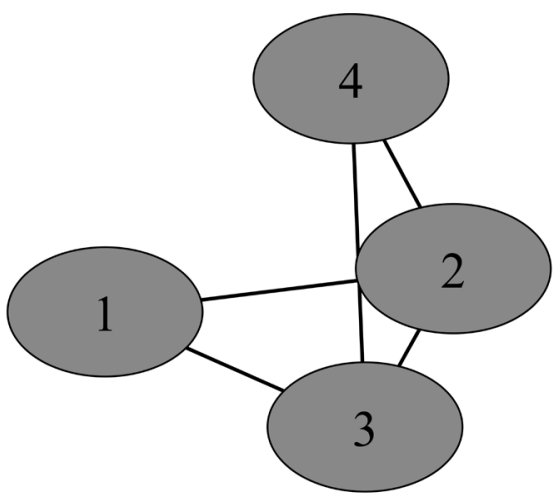

Figure 13. The DOT network as it looks in Cytoscape.

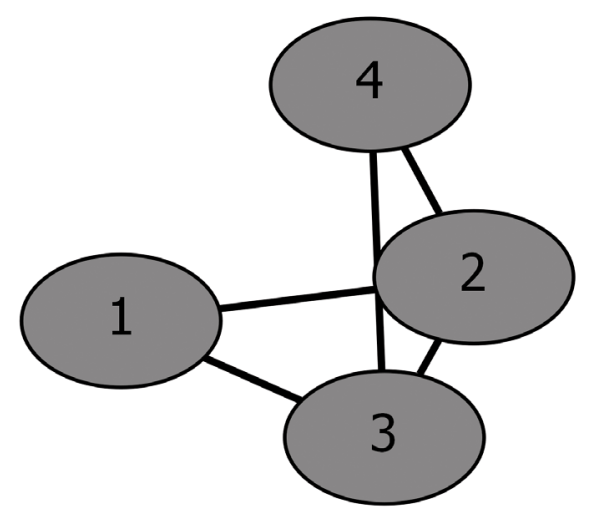

Figure 14. The PNG of the exported DOT file created with Graphviz's neato utility. 
output file. With this DOT file, we are able to use Graphviz and other programs that accept DOT files, such as NetworkX and PyGraphviz.

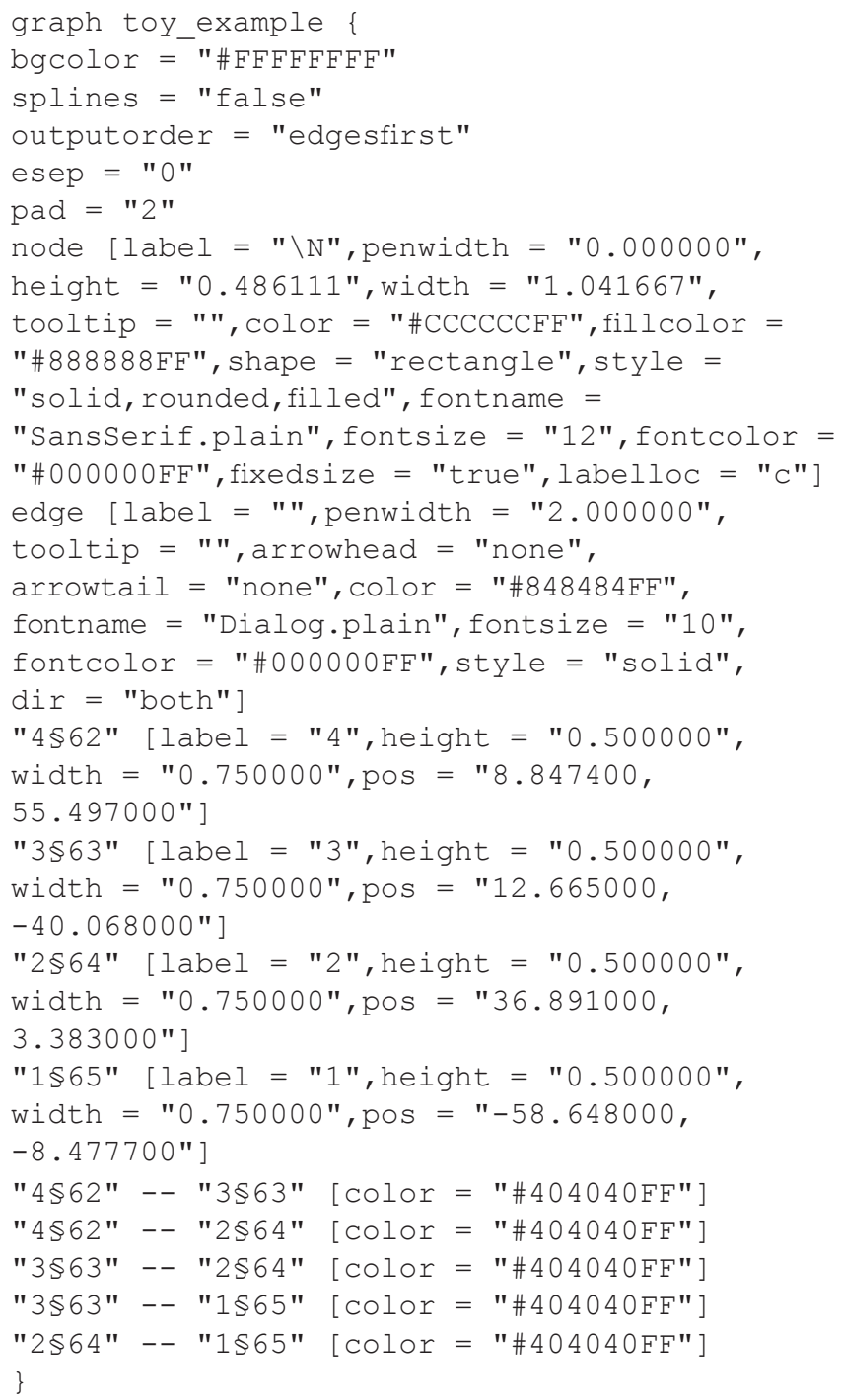

In order to prevent labels from appearing cut off, dot-app adds a padding of 2 inches to its output file. The difference in the labels' font is due to the font chosen in Cytoscape. In the output file shown above, we can see that the fontname attribute for the node default list is set to "Serif.plain". This font is not an actual font family; rather, it is one of Java's logical fonts (https://docs.oracle.com/ javase/tutorial/2d/text/fonts.html\#logical-fonts). It is a font name that the Java Runtime Environment used and that maps to a physical font. When neato encounters the font name, it attempts to find an actual font named "Serif.plain"; if it cannot find one, it uses a default font.

\section{Testing}

We verified the dot-app import and export functions separately. All of the files we used in testing are found in ZIP 2 of the Supplementary Material.

For import, we downloaded DOT files from Graphviz's gallery page (http://graphviz.org/Gallery.php) and wrote our own DOT files. We then ran Graphviz's neato utility on these files to generate DOT files that contained layout information and PNG files to use as references. We then imported the DOT files to Cytoscape and visually compared the Graphviz-created PNG files to the Cytoscape display to validate the import process of dot-app.

For export, we loaded Cytoscape test session files (https://github. com/cytoscape/cytoscape-tests/blob/master/docs/Session-Files/ Session\%20Files.md) into Cytoscape and exported individual networks from these sessions to DOT files, and then used Graphviz's neato utility to create PNG files from these. We visually compared neato's PNG output to the Cytoscape display to determine the correctness and completeness of the Cytoscape-to-DOT translation.

\section{Conclusion}

This article describes the dot-app Cytoscape app, which enables a user to import a DOT file into Cytoscape and to export a Cytoscape network as a DOT file. We demonstrated the operation of dot-app and explained its implementation and the limitations of DOT-to-Cytoscape and Cytoscape-to-DOT translation. Finally, we explained typical use cases and how dot-app delivers value in each situation.

We recognize that as long as Cytoscape and Graphviz continue to add visual shapes and features, we will need to determine if these features are found in both applications and if they are we will need to update dot-app in order to provide support for these features. Dot-app's primary goal was to allow Cytoscape to accept DOT files that represented common graph structures. We believe that dot-app as it is now can handle most of the more common DOT syntax people happen to use. However there are some, such as subgraphs, which people use that we were not able to support. We support anyone who finds dot-app lacking in some form and wishes to modify it.

\section{Software availability}

Software available from:

http://apps.cytoscape.org/apps/dotapp

Latest source code:

https://github.com/idekerlab/dot-app 
Archived source code as at the time of publication:

http://doi.org/10.5281/zenodo.8216304

License:

GNU General Public License v3

Author contributions

$\mathrm{BF}, \mathrm{ZZ}$, and $\mathrm{MM}$ were involved in writing the article. BF, ZZ, and $\mathrm{BD}$ were involved in revising the draft manuscript and have agreed on the final content. BF, ZZ, and MM were involved in designing and implementing dot-app. BD is the principal investigator for this dot-app project.
Competing interests

No competing interests were disclosed.

Grant information

This material is based upon work supported by the National Institutes of Health under Grant P41 GM103504. The grant is assigned to Dr. Trey Ideker at the University of California, San Diego (UCSD). All work was performed at UCSD.

The funders had no role in study design, data collection and analysis, decision to publish, or preparation of the manuscript.

Acknowledgements

Keiichiro Ono and Christian Zmasek helped with identifying the Cytoscape VisualProperties during the dot-app development.

\section{Supplementary Material}

Supplementary File 1: List of DOT attributes that are ignored during import.

Click here to access the data.

Supplementary File 2: Archive containing the files for the Use Cases section. Contents are:

- neato_gen_example.gv: The DOT file generated with Graphviz's neato utility

- example.cys: The Cytoscape session file that contains the example network

- cyto_gen_example.gv: The DOT file generated by dot-app for export

Click here to access the data.

Supplementary File 3: Archive containing the files for the Testing section. All networks that were used for testing along with comparison images are stored in separate folders.

Click here to access the data.

1. Shannon P, Markiel A, Ozier O, et al.: Cytoscape: a software environment for integrated models of biomolecular interaction networks. Genome Res. 2003; 13(11): 2498-504.

13(11): 2498-504.
PubMed Abstract | Publisher Full Text | Free Full Text

2. Roughan $M$, Tuke J: Unravelling graph-exchange file formats. arXiv preprint arXiv:1503.02781. 2015.

Reference Source
3. Gansner ER, North SC: An open graph visualization system and its applications to software engineering. Software Pract Exper. 2000; 30(11): 1203-1233.

Publisher Full Text

4. Fitts B, Zhang Z, Maher M: dot-app. ZENODO. 2017.

Data Source 


\section{Open Peer Review}

\section{Current Peer Review Status:}

\section{Version 2}

Reviewer Report 27 July 2017

https://doi.org/10.5256/f1000research.13068.r24109

(C) 2017 König M. This is an open access peer review report distributed under the terms of the Creative Commons Attribution License, which permits unrestricted use, distribution, and reproduction in any medium, provided the original work is properly cited.

\section{Matthias König}

Institute for Theoretical Biology, Humboldt University of Berlin, Berlin, Germany

We are satisfied that the authors have adequately addressed our comments in this new version.

Minor points

[1] Some of the test examples provided in Supplement 2 did not work with Cytoscape 3.5.1 and dot-app 0.9.4 and could not be imported

dot-app_testing/Import/ER/ER.gv : Sorry! File did not comply to dot language syntax dot-app_testing/Import/philo/philo.gv : Sorry! File did not comply to dot language syntax dot-app_testing/Import/unix/unix.gv : target node is not a member of this network

All import examples distributed with the dot-app should work with dot-app (this should be part of the unittests). If the test examples do not work with the app there will be issues with real world examples. It is stated "We verified the dot-app import and export functions separately. All of the files we used in testing are found in ZIP 2 of the Supplementary Material." This seems not to be the case.

Please fix the issue

https://github.com/idekerlab/dot-app/issues/15

[2] The app cannot be build from the provided repository after a clone.

This is reported at https://github.com/idekerlab/dot-app/issues/14

Please fix this issue. This is related to my initial review where I reported this issue.

Competing Interests: No competing interests were disclosed.

I confirm that I have read this submission and believe that I have an appropriate level of expertise to confirm that it is of an acceptable scientific standard. 
Reviewer Report 12 July 2017

https://doi.org/10.5256/f1000research.13068.r24107

(C) 2017 Lynn D et al. This is an open access peer review report distributed under the terms of the Creative Commons Attribution License, which permits unrestricted use, distribution, and reproduction in any medium, provided the original work is properly cited.

\section{David J. Lynn}

European Molecular Biology Laboratory (EMBL) Australia Group, South Australian Health and Medical Research Institute, Adelaide, SA, Australia

John Salamon

European Molecular Biology Laboratory (EMBL) Australia Group, South Australian Health and Medical Research Institute, Adelaide, SA, Australia

We are satisfied that the authors have adequately addressed our comments in this new version.

Competing Interests: No competing interests were disclosed.

We confirm that we have read this submission and believe that we have an appropriate level of expertise to confirm that it is of an acceptable scientific standard.

\section{Version 1}

Reviewer Report 20 December 2016

\section{https://doi.org/10.5256/f1000research.10513.r17222}

(C) 2016 Scardoni G. This is an open access peer review report distributed under the terms of the Creative Commons Attribution License, which permits unrestricted use, distribution, and reproduction in any medium, provided the original work is properly cited.

\section{Giovanni Scardoni}

${ }^{1}$ Center for Biomedical Computing (CBMC), University of Verona, Verona, Italy

2 Center for Biomedical Computing (CBMC), University of Verona, Verona, Italy

The article introduces an app to import/export Graphviz (.gv-.dot) files to/from Cytoscape. Since the Graphviz format is of wide usage, the app is very useful to the community.

The article is well-written and the user-guide is complete and easy to read.

As minor comments I ask more details about which characteristics can be lost passing from a format to another. I tried some network choosing them randomly from internet, and sometimes I lost features that I didn't expect to lose. 
A table explaining which features are preserved and which not will be very useful in the paper. If there are problem of space maybe the implementation details can be described in supplementary materials and not in the main paper.

The table should contains also short comments about the missing features that can be added in the future, and the problem that can be encountered implementing those characteristics that are not of immediate conversion.

I think that with these updates the paper is suitable for indexing.

Competing Interests: No competing interests were disclosed.

\section{I confirm that I have read this submission and believe that I have an appropriate level of expertise to confirm that it is of an acceptable scientific standard.}

Author Response 22 Dec 2016

\section{Ziran Zhang}

Thank you Dr. Scardoni for your comment. We will take your valuable suggestions into consideration while we are refining the article. We appreciate your effort in examining the dot-app and the article for us in detail.

Competing Interests: No competing interests were disclosed.

Reviewer Report 08 November 2016

https://doi.org/10.5256/f1000research.10513.r17125

(C) 2016 Lynn D et al. This is an open access peer review report distributed under the terms of the Creative Commons Attribution License, which permits unrestricted use, distribution, and reproduction in any medium, provided the original work is properly cited.

\section{David J. Lynn \\ ${ }^{1}$ European Molecular Biology Laboratory (EMBL) Australia Group, South Australian Health and Medical Research Institute, Adelaide, SA, Australia \\ 2 European Molecular Biology Laboratory (EMBL) Australia Group, South Australian Health and Medical Research Institute, Adelaide, SA, Australia \\ John Salamon \\ ${ }^{1}$ European Molecular Biology Laboratory (EMBL) Australia Group, South Australian Health and Medical Research Institute, Adelaide, SA, Australia \\ 2 European Molecular Biology Laboratory (EMBL) Australia Group, South Australian Health and Medical Research Institute, Adelaide, SA, Australia}


dot-app is a Cytoscape App that enables the inter-conversion of Graphviz and Cytoscape formatted files. As far as we are aware no similar application exists and this is therefore a potentially useful development.

We found no problems with the usage of the app; it integrates well into Cytoscape, and can convert back and forth between formats without issue aside from the loss of information which is documented in the article. The code is readable and very well commented.

We did have an issue when compiling from source, however. The DOT parsing jar from Alex Merz isn't being included properly when Maven builds the project.

The major concern we had was that the concept for the application is really very straight-forward and following on from this, its functionality is therefore pretty limited. We wondered initially whether there was really a need for such an application, however, dot-app has been downloaded $>1200$ times so there is obviously a market for the tool. A remaining question, however, is whether the app justifies a 10-page paper? The paper itself reads a bit more like documentation and we would have preferred to have seen a more concise paper introducing the concepts together with more detailed documentation.

We think that the paper could also benefit from a better discussion in the introduction of the motivation behind the development of dot-app. Can you give examples where one would want to create a network in one format and then convert it to another? How hard would it be to manually re-create the visualisation in Graphviz/Cytoscape?

The application also appears very reliant on the parser developed by Alex Merz (this is not referenced properly). It would be good to clarify how much of the functionality is provided by this parser and how much is extended by dot-app.

A key limitation of the app is that many of features of either format are lost when converting between them. This is because there isn't an equivalent feature in the other format. It is good that the authors are clear about which ones cannot be converted but this problem still means that manual intervention would likely be needed to customise the desired style.

One thing that could be improved is how the implicit default values are handled by dot-app. Couldn't you set reasonable values for these if they are not specified in a particular visualisation to enable more faithful conversion of the visualisation style?

One final concern is that it is very likely that the visual styles in both Graphviz and Cytoscape will continue to evolve over time. How will dot-app keep pace with these changes? Have the authors considered trying to get the Graphviz and Cytoscape communities to adopt at least the equivalent visual style features in both applications? Or at least to indicate in the Cytoscape and Graphviz documentation what is the equivalent feature (when there is one).

Minor comment:

Under Operation it would be good to start by explaining how to install the App (for novice Cytoscape users). 
Competing Interests: No competing interests were disclosed.

We confirm that we have read this submission and believe that we have an appropriate level of expertise to confirm that it is of an acceptable scientific standard, however we have significant reservations, as outlined above.

Author Response 08 Nov 2016

Ziran Zhang

Thank you Dr. David Lynn and Dr. John Salamon for your comments. We completely agree with your suggestions, and we'll add those (e.g. network conversion examples, dot-app's future adaptation, detailed installation instructions, etc.) to our article. Please expect to see the changes you recommended in the second version of our article.

Competing Interests: No competing interests were disclosed.

Reviewer Report 31 October 2016

https://doi.org/10.5256/f1000research.10513.r17148

(C) 2016 König M. This is an open access peer review report distributed under the terms of the Creative Commons Attribution License, which permits unrestricted use, distribution, and reproduction in any medium, provided the original work is properly cited.

\section{Matthias König}

${ }^{1}$ Institute for Theoretical Biology, Humboldt University of Berlin, Berlin, Germany

2 Institute for Theoretical Biology, Humboldt University of Berlin, Berlin, Germany

The article "dot-app: a Graphviz-Cytoscape conversion plug-in" is well written and describes background, implementation and use of the application in a comprehensible way.

\#\# major comments

"The differences that arise between the Graphviz network and the Cytoscape network stem from how the two programs handle implicit default values. If a DOT attribute is omitted from the DOT file when using a Graphviz utility, an implicit default value for that attribute is used. The list of DOT attributes and their default values can be found on the Graphviz website (http://www.graphviz.org/content/attrs.html)."

-> The implicit dot default values should be used as default values in dot-app for the Cytoscape styles. Users of the dot language rely on the default values and expect rendering tools to use them.

-> Especially, in combination with dot-app writing the Cytoscape default values in the exported dot this results in unnecessary changes of the graph rendering in the round trip (dot -> cytoscape -> dot). This round trip should introduce minimal changes to the rendering.

Dot default values should be used and the respective text passage and figure be updated. 
\#\# minor comments

$>>$

In the implementation all visual styles are hard coded in the visual mapping via bipasses.

It would be better to create node attributes for the dot attributes and subsequently use them in visual styles.

In this way users have access to the dot attributes, can use them in other visual mappings, create derived node attributes from them, or use them in analyses.

>> It is unclear how dot height and width are transformed to Cytoscape node height and width. There is some scaling factor, but how this is actually handled should be described in the manuscript. What information is lost in the roundtrip due to scaling.

For instance height $=$ "0.486111",width $=$ "1.041667"

result in height $>40$ in Cytoscape.

>>> The dot node font color is not rendered correctly.

See: https://github.com/idekerlab/dot-app/issues/7

>>> Saving as *.gz always adds the .dot extension so that files are called *.gz.dot.

If the user selects "name.gz" the file should be saved as 'name.gz', not as 'name.gz.dot'.

$>>$

p5 "For the import function of dot-app, we used Java-based Parser for Graphviz Documents (JPGD), a Graphviz document parser made by Alexander Merz(5)"

-> reference missing/wrong. There should be a reference for (5).

$>>$

p5 "After JPGD creates the model objects, dot-app creates a corresponding "

$\rightarrow$ something wrong with first half-sentence, probably better: "After JPGD has created the model objects, ...

$>>$

p6 "All other DOT attributes are ignored during the import process and have no effect on the visualization in Cytoscape."

If the ignored DOT attributes can be listed, state them in the manuscript as list. This is very helpful to see what information should not be used in dot files if one wants full Cytoscape support of the file. If the unsupported attributes are only the features listed in "Unsupported DOT features" than this should be clearly stated.

$>>$

p2 "The store contains nearly 300 apps that ..."

At the state of review this is already $>=300$. Add a date to the statement:

-> The store contains nearly 300 apps (October 2016) that ...

$>>$

Add reference for graphviz software/url

see for instance http://www.graphviz.org/content/citing-graphviz-paper 
The preferred citation is

@ARTICLE\{GansnerO0anopen, author $=$ EEmden R. Gansner and Stephen C. North $\}$,

title $=\{$ An open graph visualization system and its applications to software engineering $\}$, journal $=\{$ SOFTWARE - PRACTICE AND EXPERIENCE $\}$, year $=\{2000\}$, volume $=\{30\}$, number $=\{11\}$, pages $=\{1203--1233\}$

\}

and the URL is www.graphviz.org

$>>$

p8 use cases

Provide the *.gv files and Cytoscape files of the use cases in the supplement.

This will provide the necessary materials to follow the provided examples. I tried to save the files from the pdf which did not work (due to line breaks in pdf), copying the examples from the HTML version of the manuscript worked. Adding the two example files to the supplementary information will improve this.

$>>$

p9 testing

Provide the url/repository for the test files and test results.

There should be a page which shows all the comparison images with the respective dot files. In the article it is mentioned that this exists, but no resource is given for the test files and test results.

$>>$

p9 conclusion

"This article describes the dot-app Cytoscape app, which enables a user to import a DOTformatted **here** into Cytoscape"

-> word missing

$>>$

p9 conclusion

"we recognize that future versions of Cytoscape may introduce new visual effects"

-> formulation. Better: 'visual shapes and styles", visual effects are something else.

$>>$

p9 conclusion

"we recognize that future versions of Cytoscape may introduce new visual effects (e.g., new arrow heads) that present opportunities for rendering DOT files more truly or for the loss of formatting information if the DOT format cannot represent them."

-> second half of sentence does not make sense (... or for the loss of formating). Please reformulate to clarify what is meant.

$>>$

The github release corresponding to the zenodo code is missing. The latest github release is 0.9.1 on 11.September, the zenodo was created in February. This makes it difficult to try to build the 
app from the mentioned source code, by checking out a corresponding tag.

Which version is packed in zenodo? 0.9.2?

Also the current app version in the app store is 0.9 .3 , but there is no 0.9 .3 release on github.

Please create a github release which is corresponding to the zenodo release and the mentioned code state of the publication.

$>>$

I was unable to build the app from latest source code. The app is working via the app store, but in addition it should be possible to build the latest source code.

https://github.com/idekerlab/dot-app/issues/8

git clone https://github.com/idekerlab/dot-app.git

mvn clean install

results in

[INFO] Compiling 16 source files to /home/mkoenig/git/dot-app/target/classes

[INFO]

[INFO] BUILD FAILURE

[INFO]

[INFO] Total time: $7.250 \mathrm{~s}$

[INFO] Finished at: 2016-10-31T12:48:57+01:00

[INFO] Final Memory: 21M/238M

[INFO]

[ERROR] Failed to execute goal org.apache.maven.plugins:maven-compiler-plugin:2.0.2:compile (default-compile) on project dot-app: Compilation failure: Compilation failure:

[ERROR] /home/mkoenig/git/dotapp/src/main/java/org/cytoscape/intern/read/reader/NodeReader.java:[46,36] error: package com.alexmerz.graphviz.objects does not exist

[ERROR]

$\cdots$

This should be fixed, I would recommend to setup some Continous Integration with travis to make sure the latest version always builds.

see https://github.com/idekerlab/dot-app/issues/8

Competing Interests: No competing interests were disclosed.

I confirm that I have read this submission and believe that I have an appropriate level of expertise to confirm that it is of an acceptable scientific standard, however I have significant reservations, as outlined above.

Author Response 31 Oct 2016

Ziran Zhang

Thank you so much Dr. Matthias König for your insightful comments. We really appreciate your efforts of carefully testing the dot-app features and throughly examining our article. 
We thank you for bringing the problems you found to our attention. While waiting for another referee's upcoming peer review report, we will take a close look into the issues and address them for our next version.

Competing Interests: No competing interests were disclosed.

Reviewer Report 27 October 2016

https://doi.org/10.5256/f1000research.10513.r17124

(C) 2016 Bonnet E. This is an open access peer review report distributed under the terms of the Creative Commons Attribution License, which permits unrestricted use, distribution, and reproduction in any medium, provided the original work is properly cited.

\section{Eric Bonnet}

${ }^{1}$ Institute of Genomics, CEA (French Alternative Energies and Atomic Energy Commission), Evry, France

${ }^{2}$ Institute of Genomics, CEA (French Alternative Energies and Atomic Energy Commission), Evry, France

This article describes a new Cytoscape plugin called "dot-app" designed for the import and export of Graphviz files conforming to the DOT format for representing graph topologies, layouts and formatting.

Graphviz is a popular set of open source software tools initiated at the AT\&T Labs Research that can generate or process DOT files. Many other software tools are using Graphviz to represent all kinds of graphs.

The DOT language support visual features and attributes that are not currently included by Cytoscape network formats. There is currently no Cytoscape plugin for the import and export of networks to the DOT language. Therefore the dot-app plugin may be useful to convert and import biological networks, and might be very interesting for the large community of Cytoscape users.

The article is globally clear, well-organized and logically structured. I particularly appreciated the efforts of the authors to explain the details of the conversion process and exactly what kind of visual properties are imported and exported, including the properties that are not supported.

I would suggest minor improvements to the article that could make it more precise and valuable for the readers.

1. Add a table for at least some of the main visual features and attributes supported by the DOT format and indicate which ones are supported by XGMML/GraphML. The sentence mentioning the differences in the introduction section is too vague.

2. Compile also in a small table some examples of applications (related to biology or not) that produces or consumes DOT files. Here also the paragraph discussing this in the 
introduction section is quite vague.

Minor comments:

The reference to the JPGD parser do not appear in the bibliography.

A few sentences on how to install the app in Cytoscape might be helpful.

Competing Interests: No competing interests were disclosed.

I confirm that I have read this submission and believe that I have an appropriate level of expertise to confirm that it is of an acceptable scientific standard.

\section{Author Response 30 Oct 2016}

\section{Ziran Zhang}

Thank you Dr. Bonnet for your valuable comments. We will look into the possible improvements and minor issues you brought up, and fix them in our next version. Again, we really appreciate your detailed observations!

Competing Interests: No competing interests were disclosed.

The benefits of publishing with F1000Research:

- Your article is published within days, with no editorial bias

- You can publish traditional articles, null/negative results, case reports, data notes and more

- The peer review process is transparent and collaborative

- Your article is indexed in PubMed after passing peer review

- Dedicated customer support at every stage

For pre-submission enquiries, contact research@f1000.com 